\title{
Factors that Determine the Magnitude and Time Course of Human H-Reflexes in Locomotion
}

\author{
M. Edamura, J. F. Yang, and R. B. Stein \\ Division of Neuroscience and Department of Physiology, University of Alberta, Edmonton, Canada T6G 2S2
}

The soleus H-reflex amplitude is deeply modulated during locomotion in humans (Capaday and Stein, 1986). Moreover, at a constant stimulus intensity, the slope of the relationship between the amplitude of the soleus $\mathrm{H}$-reflex and the background electromyogram (EMG) changes with different locomotor tasks (Capaday and Stein, 1987a). Two further aspects are studied here. First, we recorded the reflex during overlapping speeds of walking (2.0-7.5 $\mathrm{km} / \mathrm{hr})$ and running (5-9 km/hr) to determine whether the speed, the motor output, or the form of locomotion was most important in setting the slope of this relationship between H-reflex and background EMG. Second, we determined the time course of change in the H-reflex amplitude and the possible site of action for the reflex depression during the transition from standing to walking. The primary determinant of the slope was found to be the form of locomotion. The differences between running and walking could not be explained entirely by either movement speed or motor output. For walking, the slope varied inversely with the speed and the motor output of locomotion. This compensation in slope as a function of motor output may prevent saturation of the motoneuron pool. The appropriate reflex amplitudes for a particular locomotor pattern are activated rapidly and completely within a reaction time, and simultaneously with the activation of muscle activity for the initiation of walking. Mechanisms for the rapid change seen during the initiation of locomotion most likely act presynaptically on the muscle spindle afferents. The time course and magnitude of this change are correlated with the activity of the tibialis anterior muscle.

Spinal reflexes are modulated to adapt motor programs to changing conditions, but the mechanisms underlying this modulation are still uncertain. Even the simplest reflex, the monosynaptic stretch reflex between primary muscle spindle (group Ia) afferents and alpha motoneurons, can be modified through the action of gamma motoneurons on the receptors, by presynaptic inhibition of the sensory terminals, or by postsynaptic mechanisms acting on the alpha motoneurons. Electrical stimulation of the group Ia afferents was first shown by Hoffmann (1918) to elicit a monosynaptic reflex and is now referred to as

\footnotetext{
Received Jan. 25, 1990; revised Sep. 10, 1990; accepted Sep. 13, 1990.

Support for the research was provided by the Medical Research Council of Canada. M. Edamura and J. F. Yang were postdoctoral fellows of the Rick Hansen Man in Motion Fund and the Alberta Heritage Foundation for Medical Research, respectively.

Correspondence should be addressed to Dr. R. B. Stein, Division of Neuroscience, 513 Heritage Medical Research Bldg., University of Alberta, Edmonton, Canada T6G 2S2.

Copyright (C) 1991 Society for Neuroscience $0270-6474 / 91 / 110420-08 \$ 03.00 / 0$
}

the H-reflex. This electrical analog of the stretch reflex eliminates the effects of gamma motoneurons.

The H-reflex is strongly modulated in a number of human muscles during cyclical movements as a function of the time in the movement cycle (Capaday and Stein, 1986, 1987a; Crenna and Frigo, 1987; Boorman et al., 1989; Collins et al., 1989; Dietz et al., 1990). Moreover, the relation between the H-reflex amplitude and the background electromyogram (EMG) is very different for different tasks such as standing, walking, and running (Capaday and Stein, 1986, 1987a). This reflex is highest in standing, substantially lower in walking, and lower still in running. For clarity, we will refer to the cyclical modulation of the reflex amplitude within a locomotor task as "time dependent," and the different reflex amplitudes achieved under different locomotor conditions as "task dependent." This paper is primarily concerned with the task-dependent changes in reflex amplitude.

The relationship between the reflex amplitude and the level of the background EMG can often be fitted by a straight line (e.g., Fig. $2 A, B, C$ ). The differences between walking and running consist largely of a change in the slope relating these 2 variables (Capaday and Stein, 1987a). Since the input is approximately constant and the output varies, the difference in slope represents a modulation in central reflex gain that is distinct in the 2 forms of locomotion (Capaday and Stein, 1987b). Changes in the slope will be referred to here as changes in reflex gain for simplicity, although this is an operational definition of reflex gain rather than one derived rigorously from an input-output relationship.

What are the factors that determine the task-dependent reflex gain? What mechanisms does the CNS use in setting reflex gain? What are the dynamics with which reflexes are modified when the task is changed? These questions form the subject of this study.

\section{Materials and Methods}

Details of the experimental procedures and the data analysis methods have been described elsewhere (Capaday and Stein, 1986, 1987a) and are summarized briefly here. Modifications made in the present experiments will also be described.

\section{Eliciting and recording the soleus $H$-reflex}

The EMG of the soleus (SOL) and the tibialis anterior (TA) muscles were recorded with surface silver/silver chloride electrodes (Beckman type, 8-mm diameter) in a bipolar configuration, after reducing the skin impedance by light abrasion and cleaning. The myoelectric signal was bandpass filtered (10-10 kHz) to eliminate artifacts. The same type of electrode was used as a cathode to stimulate the tibial nerve in the popliteal fossa. The electrode was placed over the tibial nerve in a position that optimized the $\mathrm{H}$ - and $\mathrm{M}$-waves of the SOL, and was taped to the skin. The anodal electrode was placed above the patella. The stimuli ( 1 msec duration) were delivered randomly to the tibial nerve 
by an isolated, constant-voltage stimulator (interstimulus intervals 1 $2 \mathrm{sec}$ ). The stimulus intensities used generated a small direct motor response (M-wave) and a near maximal $\mathrm{H}$-reflex (i.e., near the plateau phase of the H-reflex recruitment curve).

\section{Experiments on different walking and running conditions}

In the first series of experiments, we determined whether the speed, the motor output, or the form of locomotion was most important in setting the task-dependent reflex gain. If the form of locomotion is most important, then one might expect that the gain would be the same at different speeds of walking, but would differ hetween walking and running at the same speed. On the other hand, if gain depends on the speed of movement (or motor output), it might be the same in different forms of locomotion at the same speed, and change gradually as speed (and motor output) varied in both gait patterns. H-reflexes of the SOL muscle were obtained from 6 human subjects during various speeds of walking $(2.0-7.5 \mathrm{~km} / \mathrm{hr})$ and running $(5.0-9.0 \mathrm{~km} / \mathrm{hr})$ on a treadmill. A single subject performed 2 or more different kinds of locomotor tasks in 1 day. A total of 37 conditions were studied.

The H-reflexes were averaged based on the time in the step cycle at which they were elicited. The beginning of a step cycle was defined as the time when a switch placed on the heel of the shoe was closed by contact with the ground. The latency between the step marker and the stimulus marker was used to determine in which of the 16 phases of the step cycle the stimulus occurred. Those responses that occurred in the same phase were averaged together (usually 10-20 sweeps). Within a phase, the $\mathrm{M}$ - and $\mathrm{H}$-waves varied little from sweep to sweep (standard error 10-20\%, see Fig. 2C).

To acquire H-reflexes evoked by the same stimulus strength at each phase of the step cycle, the corresponding M-waves were used as indicators of the effective stimulus strength (Capaday and Stein, 1986). The $\mathrm{M}$-waves were closely matched across the 16 phases of the step cycle (varying less than $30 \%$ ) by repeating the experiment at various stimulus intensities. Thus, $\mathrm{H}$-reflexes occurring at each of the 16 phases of the step cycle could be compared at a stimulus intensity that excited the same number of motor axons and presumably the same number of sensory axons. Although there are substantial changes in the muscle length and shape during movement which could change the $\mathrm{M}$-wave recorded (Myklebust et al., 1984), these changes result in little variation in the $\mathrm{M}$-wave and do not modify the H-reflex amplitude substantially (Llewellyn, 1989). Because the experiments generally required 4-6 hr to complete, control trials were interspersed in which the $M$ versus $H$ relationship was verified under static conditions. A constant $\mathrm{M}$ to $\mathrm{H}$ relationship was a further check that the stimulating and recording conditions were relatively constant throughout the experiment.

The EMG activity associated with the undisturbed locomotor cycle was averaged on-line after full-wave rectification and low-pass filtering (30-Hz RC filter). The averaging was triggered by the contact switch placed under the heel. This procedure was repeated at the beginning and the end of an experiment to ensure that the pattern of activity remained the same throughout the duration of the experiment.

To examine the reflex gain, the peak-to-peak amplitude of the $\mathrm{H}$-reflex $(\mathrm{mV})$ was plotted as a function of the mean EMG level $(\mathrm{mV})$ during the phase in which the reflex occurred, and the slope of the least-squares fitted line $(\mathrm{mV} / \mathrm{mV})$ was calculated. The relationship between the H-reflex and background EMG can be defined unambiguously only when there is a nonzero level of EMG activity. Therefore, in calculating the regression line we only used data obtained during the stance phase and the first time segment of the swing phase when the EMG level is declining toward zero.

\section{Experiments on the transition from standing to walking}

Since the reflex gain is dramatically different between standing and walking (Capaday and Stein, 1986), how is the change brought about? This can be studied by examining the transition in the reflex from the high value in standing to the low value in walking. $\mathrm{H}$-reflexes were elicited in 4 subjects while they went from a quiet standing posture to walking. Subjects were instructed to start walking as soon as they heard an auditory signal, and to lead with the left leg. Generally, subjects took 4-5 steps before stopping to prepare for the next trial. Reflexes were elicited randomly from the trailing leg (i.e., right leg). The reflexes were studied in this leg because the contralateral leg enters into the swing phase early. The very low SOL EMG and H-reflex in the swing phase could interfere with the determination of the relationship between the reflex and SOL EMG. Moreover, the trailing limb remains relatively stationary and the SOL remains minimally active for nearly $1 \mathrm{sec}$ after the auditory tone, until the push-off phase is initiated. This background activity is important because it reflects the excitability of the motoneuron pool during the performance of the task. Changes observed in the H-reflex can then be attributed either to postsynaptic changes of the SOL motoneuron pool (as reflected by the background activity) or to presynaptic factors independent of the excitability of the motoneuron pool.

Reflexes were averaged based on the time at which they were elicited with respect to the auditory start signal. Generally, the reflex changes were followed for the duration of approximately $1 \mathrm{sec}$ from the time of the start signal. This $1-\mathrm{sec}$ time period was divided into 16 equal segments and those reflexes that occurred within the same segment were averaged together (approximately 10 sweeps per segment).

Changes in reflex gain seen during the initial step were compared with those during normal treadmill walking for the same subject. Similarities between these conditions indicated whether the early changes in reflex gain were representative of the normal walking pattern.

The EMG patterns associated with the initiation of walking were averaged on-line from the time of the start signal. It was clear that a large TA burst was the first consistent activity found in all subjects. To determine whether this TA burst was important in modifying the SOL $\mathrm{H}$-reflex, experiments were performed in which subjects started from a forward lean while supported by a chair. This starting position reduced the TA burst while the SOL remained relatively unchanged.

Experiments were also performed to determine the strength of reciprocal inhibition from the TA under static conditions. Subjects tried to mimic the background activation levels of the muscles during the initiation of walking, by cocontracting the SOL and TA statically. H-reflex depression elicited with increasing levels of TA activation indicated the strength of reciprocal effects from the TA under static conditions.

\section{Results}

\section{Determinants of $H$-reflex gain during locomotion}

Figure 1 shows the pattern of EMG activity generated in the SOL of a normal human subject walking and running on a treadmill, both at $6 \mathrm{~km} / \mathrm{hr}$. Each trace begins at the time of heel contact which initiates the stance phase. The SOL activity increases during the stance phase, and more or less coincides with the duration of the stance phase. Electrical stimuli applied to the tibial nerve elicit a small $\mathbf{M}$-wave from direct stimulation of motor axons and a reflex $\mathrm{H}$-wave from stimulation of muscle spindle afferents.

Various stimulus strengths were used and levels were chosen at each part of the step cycle that stimulated an approximately constant fraction of motor axons (the M-wave showed no significant trend, but varied randomly with a standard deviation of $16 \%$ across the different phases of walking and running in Fig. 1). Presumably, an approximately constant fraction of Ia muscle spindle afferents were also stimulated. Even with a constant affercnt input, the reflcx $\mathrm{H}$-wave incrcascd in parallel with the increase in SOL voluntary activity, as previously shown (Capaday and Stein, 1987a). Note that the H-reflex never reached as high values during running as during walking, though the peak EMG activity was $19 \%$ greater during running.

This difference between walking and running is seen more clearly in Figure 2, $A$, in which the H-reflex is plotted against the mean EMG level in each part of the step cycle. Although more complex curves could be used, the data are well fitted by a straight line (linear correlation coefficient of 0.90 and 0.76 for walking and running, respectively). The slopes of the straight lines are $12.5 \pm 2.0$ for walking, and $3.7 \pm 1.4$ for running (mean $\pm \mathrm{SE}$ ), which are significantly different at the 0.005 level of confidence. The intercepts were $0.68 \pm 0.39$ and $0.59 \pm 0.40$, respectively, which were not significantly different from zero or from each other. The differences in slope clearly do not result 
Figure 1. Comparison of SOL H-reflex and background EMG pattern in 1 subject walking and running at same speed $(6 \mathrm{~km} / \mathrm{hr})$ on same day. The SOL EMG rises in a ramplike fashion through the stance phase and is silent in swing phase during both locomotor tasks. The peak SOL EMG achieved is slightly higher in running. Each tracc represents the average of 50 steps. The H-reflex elicited from the SOL rises and falls more or less in parallel with the background SOL EMG. The modulation in the H-reflex amplitude (filled circles) is seen despite a constant effective stimulus strength through the step, as reflected by the relatively constant M-wave amplitude (open circles). Moreover, the average M-wave amplitude (solid line through the open circles) was well matched for both tasks.
Wolking
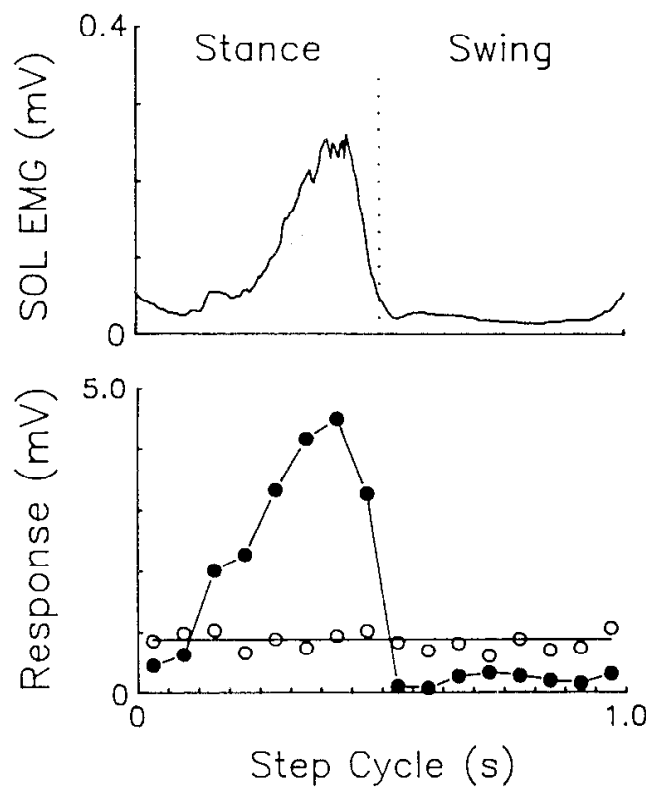

Running


from saturation of the motoneuron pool, because the reflex amplitude is lower for running at all levels of background EMCr, as previously shown (Capaday and Stein, 1987a). Similar results were seen for a second subject on 2 different days (Fig. 2B,C) in which the data were well fitted with straight lines. The variability of the H-reflex from stimulus to stimulus (SE bars in Fig. $2 C$ ) and from day to day (compare Fig. $2 B$ with $2 C$ ) were quite small.

This difference in slope was noted previously (Capaday and Stein, 1987a), but in the previous study, a comparison was made between walking at $4 \mathrm{~km} / \mathrm{hr}$ and running at $8 \mathrm{~km} / \mathrm{hr}$. Thus, speed and motor output, as well as type of locomotion, varied and the relative importance of these factors could not be determined. Figure 2, $D$, in which the slope of the relationship between background EMG and SOL H-reflex is plotted as a function of locomotor speed for the subject of Figure 2, $B$ and $C$, illustrates that the form of locomotion is the important determinant. This subject also walked at $3 \mathrm{~km} / \mathrm{hr}$ and ran at 9 $\mathrm{km} / \mathrm{hr}$ on the same day. There is some decrease in slope at the faster walking speed, but both values are a factor 3 or 4 times higher than the values for running.

Six normal subjects ran and walked at the same speed. Speeds 5-7 km/hr were used for different subjects, depending on their preference. The relation between H-reflex and mean EMG in running had values of slope only $56.6 \pm 10.9 \%$ of those in walking at the same speed. The slope value, averaged across subjects, was significantly lower in running than in walking at a probability level less than 0.05 (paired $t$ test).

The EMG levels and slopes on different days and between subjects were substantially different. To compare different sub-
Figure 2. H-reflex amplitudes obtained during walking and running at same speed of $6 \mathrm{~km} / \mathrm{hr}$ (from same subject as in Fig. 1). $A$, H-reflex amplitudes plotted against the average background activation level of the SOL at the corresponding times in the step cycle. The best-fitting regression lines for the 2 tasks have similar intercepts but different slopes, indicating that the reflex gain was lower in running than in walking. $B$ and $C$, Similar results were obtained from another subject walking and running at $6 \mathrm{~km} / \mathrm{hr}$ on 2 different days. $D$, Slope of the regression lines for 4 different locomotor conditions in this subject, all measured with the electrodes in the same position on 1 day, clearly shows that the reflex gains associated with running were lower than those associated with walking. Standard error bars were computed for the $\mathrm{H}$-reflexes in $(C)$ and for the slopes in $(D)$.
A
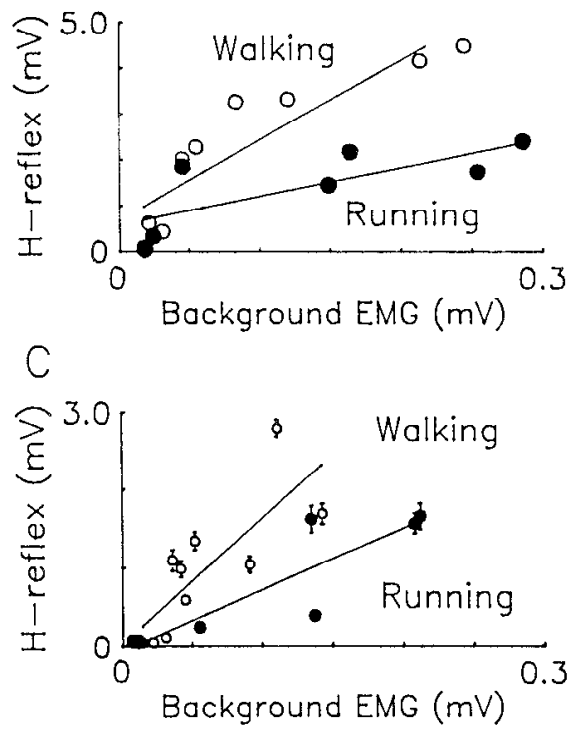

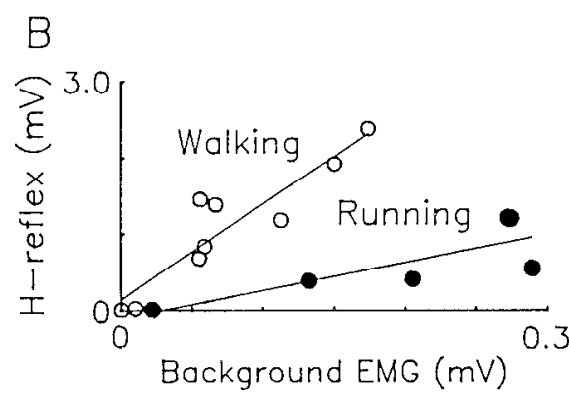

$D$

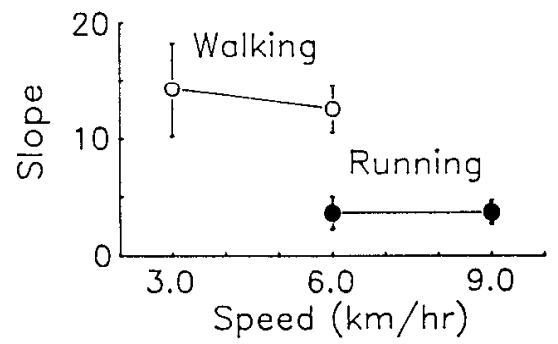



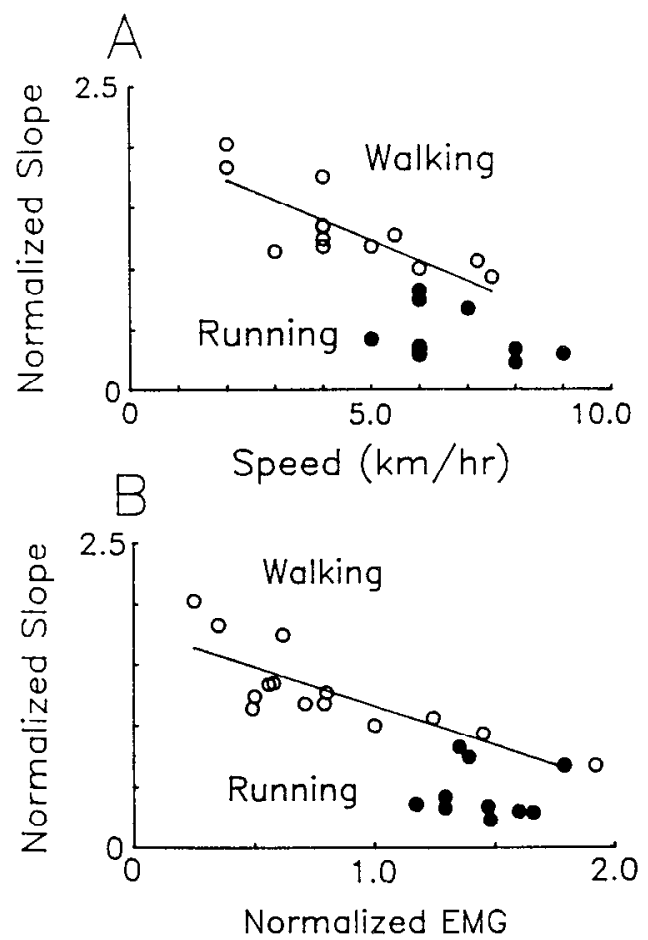

Figure 3. Slope of relationship between H-reflex amplitude and background SOL EMG for all subjects in walking (open circles) and running (filled circles) normalized to values at $6 \mathrm{~km} / \mathrm{hr}$ walking (as described in the text). $A$, This slope is a function of locomotor speed in walking, as reflected by the strong linear correlation (solid line). The values for running were all lower than walking and generally more variable. The slope of the regression line (not shown) for running was not significantly different from zero. Locomotor speed is clearly not the only factor that determines the reflex gain. $B$, The slope is also correlated with the peak SOL EMG obtained in walking. Again, the slopes obtained in running were all lower than those in walking, suggesting that background EMG is not the only factor that determines the reflex gain. Both $A$ and $B$ suggest that reflex gain is largely a function of the locomotor task. jects, the slope at $6 \mathrm{~km} / \mathrm{hr}$ was normalized to 1 . The slopes in 3 subjects, who walked at both 4 and $6 \mathrm{~km} / \mathrm{hr}$, were on average $35 \%$ greater at $4 \mathrm{~km} / \mathrm{hr}$. Thus, for another 3 subjects who walked at 4 , but not $6 \mathrm{~km} / \mathrm{hr}$, the values were normalized to 1.35 for $4 \mathrm{~km} / \mathrm{hr}$ and in 1 subject who walked at 5 , but not $6 \mathrm{~km} / \mathrm{hr}$, to 1.18 at $5 \mathrm{~km} / \mathrm{hr}$. With this normalization the values for 10 different running and 10 different walking conditions could be compared over a $2-9 \mathrm{~km} / \mathrm{hr}$ range of speeds.

The results are shown in Figure $3, A$. There was a significant trend for the slope to decrease with increasing speed during walking (correlation coefficient $r=-0.82$, variance accounted for significantly different from zero at $p<0.05, F$ test; Pedhazur, 1982). A similar trend was evident during running, but the data were more scattered and the trend was not significant $(r=-0.41)$. The slopes obtained during running were a fraction of that predicted for walking by the regression line (average fraction: 0.47 \pm 0.20 ) and all fell below the regression line. The probability of this happening is less than 0.001 (run-test, Feller, 1957).

However, higher EMG levels are reached during running than during walking at the same speed. The reflex gain could conceivably be controlled by the EMG level, rather than the task. To test this possibility the data were replotted against the peak EMG level reached in each condition. The EMG values were also normalized to 1 for walking at $6 \mathrm{~km} / \mathrm{hr}$. In those subjects who walked at both 4 and $6 \mathrm{~km} / \mathrm{hr}$, the normalized peak EMG level was on average 0.58 at $4 \mathrm{~km} / \mathrm{hr}$, so this value was used to normalize data from subjects who walked at 4 , but not $6, \mathrm{~km} /$ $\mathrm{hr}$ and a value of 0.79 was used to normalize the data from the 1 subject who walked at 5 , but not $6, \mathrm{~km} / \mathrm{hr}$.

As seen in Figure 3, $B$, the trend for the slope to decrease at increasing EMG levels during walking is even tighter $(r=-0.89)$ than that for slope to vary with speed. Again, the values for running are more scattered and the trend was not significant ( $r$ $=-0.02$ ). Nonetheless, 9 of the 10 values for running fall below the trend line for walking; the probability of this happening is less than 0.01 (run-test, Feller, 1957). This result further sup-
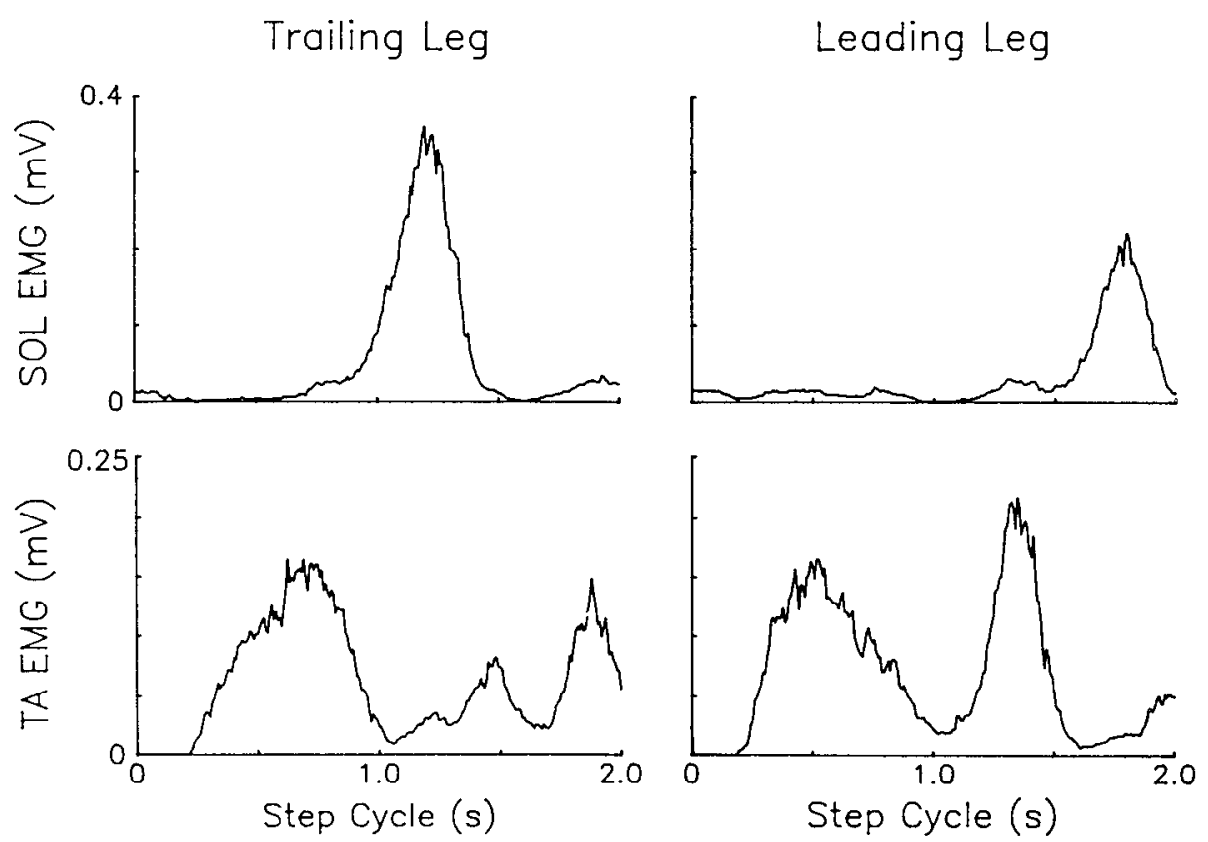

Figure 4. EMGs of SOL and TA muscles show characteristic patterns during initiation of walking. All traces begin at the time of the auditory tone to the subject. The pattern for quiet standing remains unchanged for at least $100 \mathrm{msec}$ and is characterized by low levels of SOL activity. At approximately 200 msec (in this subject), a large burst of TA EMG is seen bilaterally, with a concurrent decrease in the SOL EMG. In the leading leg, a second TA burst initiates the swing phase while concurrently, in the trailing leg, a SOL burst generates the first push-off phase. This is followed by the typical SOL and TA EMG patterns of normal walking. 
Figure 5. Slightly different EMG patterns of trailing leg observed in different subjects. Subject \#2 exhibited a pattern similar to that shown in Figure 4, while Subject \# 1 showed a higher level of SOL activity. H-reflexes elicited from the SOL were initially high as in standing, then decreased dramatically within 200 msec from the time of the auditory tone (time zero). The change in reflex amplitude occurred despite the constant stimulus strength, as reflected by the M-wave. The time course of the reflex change appeared to be negatively correlated with the time course of the TA activity.
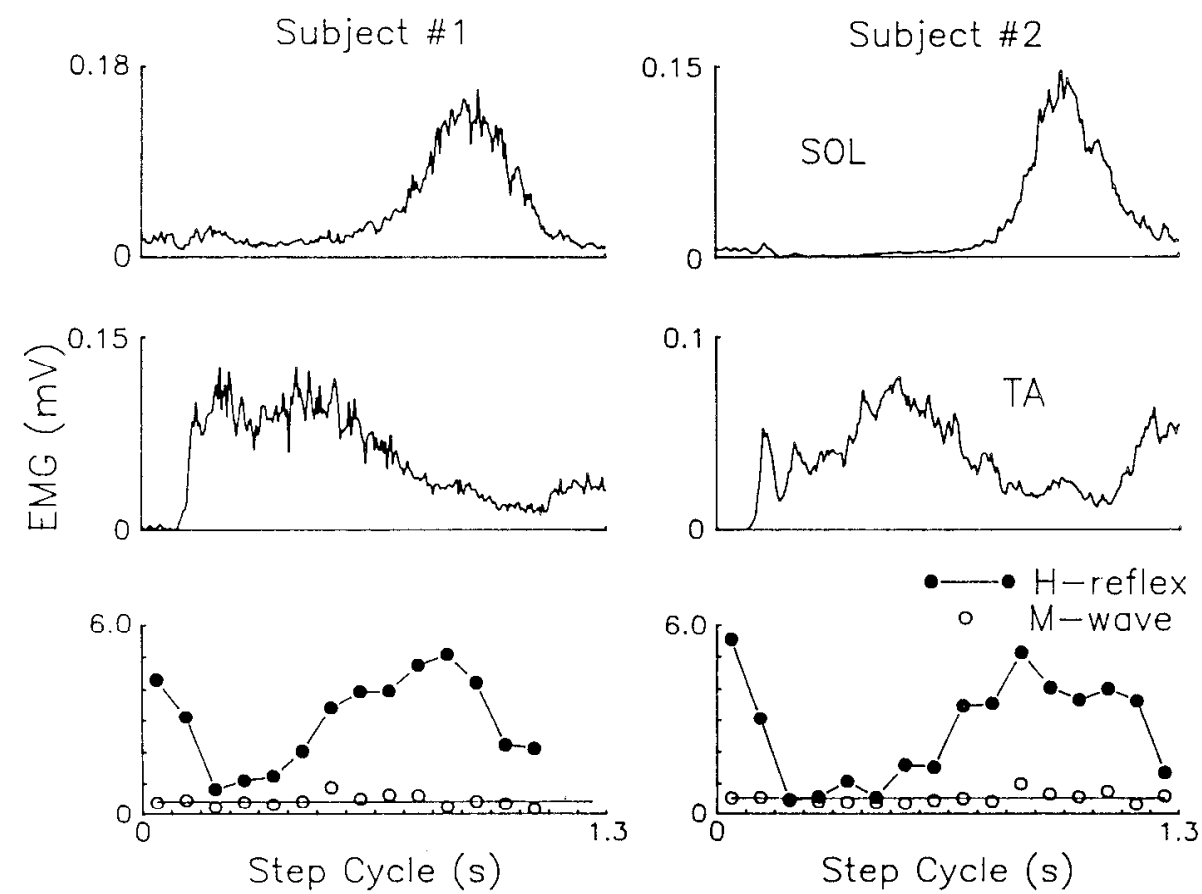

ports the idea that the reflex modulation is set differently for running than for walking, even after differences in speed and EMG levels are taken into account.

\section{The initiation of walking}

Quiet standing is usually characterized by very low levels of $\mathrm{SOL}$ contraction and very high $\mathrm{H}$-reflexes. The transition from standing to walking is achieved by a large TA burst bilaterally at approximately $200 \mathrm{msec}$ (average reaction time) after the start signal (Fig. 4), presumably to pull the body forward. The SOL EMG decreases simultaneously with the initiation of the TA burst in some subjects. The TA burst is prolonged (for approximately $700 \mathrm{msec}$ ) bilaterally and decreases as the body rotates forward over the foot. Then, in the leading leg, a second TA burst initiates the first swing phase in parallel with the first pushoff of the trailing leg from its SOL burst. Finally, the trailing leg begins its first swing phase, with the characteristic double burst pattern seen in human TA, accompanied by a push-off on the other leg.

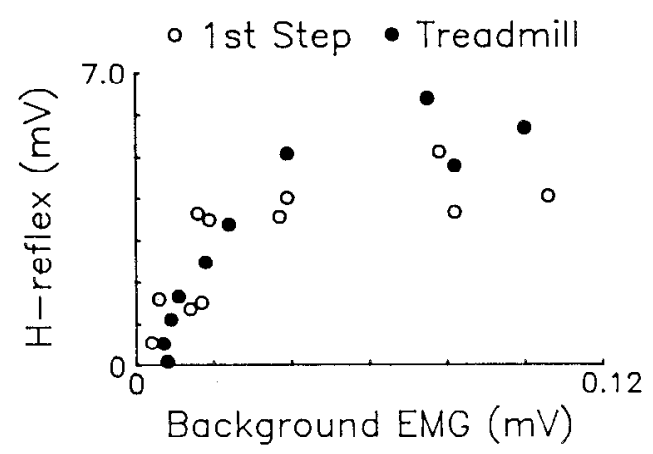

Figure 6. Relationship between H-reflex amplitude and background SOL EMG was similar for first step (open circles) and for normal treadmill walking (filled circles). The reflex gain appears to be switched to the value observed in steady state walking within the first step.
The SOL H-reflex is modulated at the initiation of walking as shown in Figure 5 for 2 subjects. The H-reflex amplitude during the first $100 \mathrm{msec}$ after the auditory tone is high, and is representative of the reflex amplitude in standing, as shown by averages obtained prior to the tone (not shown). A sudden depression of the reflex occurs within $200 \mathrm{msec}$, coincident with the rise in TA EMG and the slight depression of SOL activity. The depression in the reflex extends approximately 400-600 msec, relatively independently of the SOI activation pattern (e.g., Subjects \#1 and \#2 in Fig. 5), but the depression seems to be inversely correlated with the activity of the TA EMG. Eventually, the reflex slowly rises, either in parallel with, or slightly in advance of, the SOL burst of push-off. All subjects exhibited this dramatic reflex depression within the reaction time, although the time course of the later rise in the reflex differed slightly between subjects (e.g., Subject \#1 versus $\# 2$ in Fig. 5).

The reflex during the first step was compared with that during maintained walking on a treadmill, to determine whether the dramatic depression in the first step might be related to conditions unique to that step. One of the obvious differences between the 2 conditions is the large initial TA burst, which occurs only in the first step. The H-reflexes of the first step were similar to those of normal treadmill walking at $4 \mathrm{~km} / \mathrm{hr}$ (Fig. 6), so thc reflex depression seen in the first step represents a rapid and complete switch to the reflex gain for walking.

Modification of the starting position can dramatically change the amplitude of the initial TA burst. When subjects leaned forward holding on to a chair, the background activation level of the SOL could be maintained at much the same level as in the normal transition task while the TA burst was largely eliminated (Fig. 7). Under these conditions, the reflex depression was still present, though to a lesser extent.

The importance of the reciprocal effect from the TA was further demonstrated in 2 experiments. Under static conditions, different degrees of TA contraction were produced with a level of SOL EMG approximating the levels seen during the transi- 

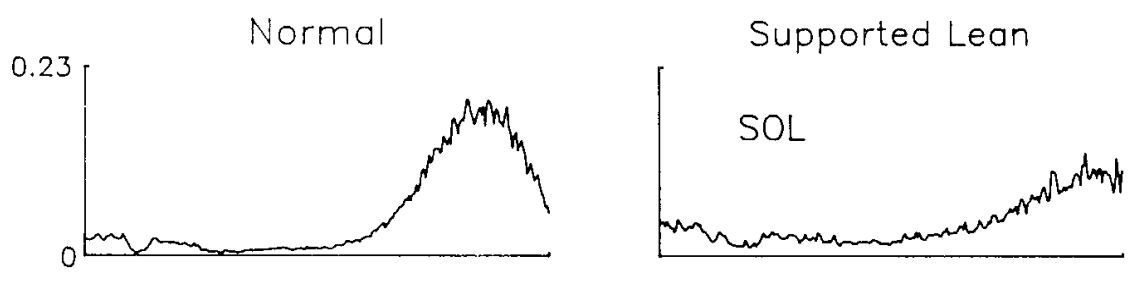
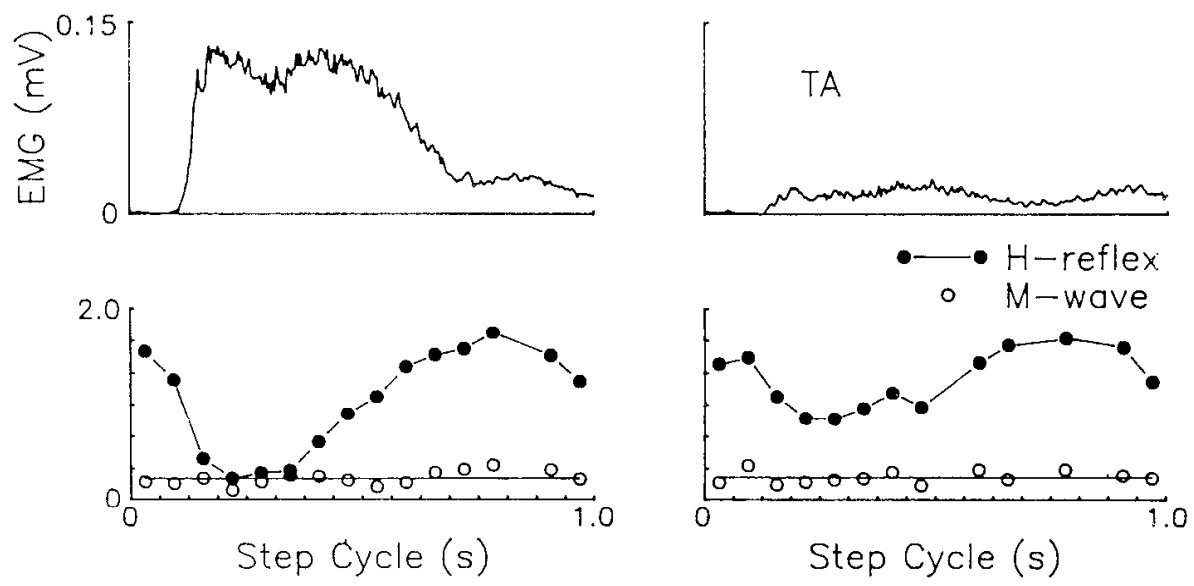

Figure 7. Starting subject from a forward lean while supported by a chair (supported lean) largely eliminated the large TA burst associated with normal initiation of walking. The SOL activation pattern was largely unchanged by this maneuver at least for the first 500 msec. The depression in the SOL H-reflex normally seen during the initiation of walking was considerably less dramatic when the TA burst was reduced, though the reflex depression could not be eliminated. The stimulus intensity was constant for the duration of both tasks, as reflected by the relatively constant M-wave amplitude. tion task. The reciprocal effects were strong under both static and dynamic conditions, with a stronger effect seen during the dynamic transition (Fig. 8).

\section{Discussion}

The H-reflex amplitude of the SOL muscle appears to be determined primarily by the form of locomotion. A task-dependent change in the reflex gain occurs rapidly at the time locomotion is initiated. This new gain is representative of that normally seen in steady state walking, suggesting that the appropriate reflex gain is elicited rapidly at the start of the movement. The following discussion suggests that the site of action for the task-dependent gain change is presynaptic and correlated with the activity of the TA.

\section{What are the determinants of reflex gain in locomotion?}

The form of locomotion largely determines the gain of the SOL $\mathrm{H}$-reflex. If the speed of locomotion were the primary determinant of the reflex gain, then one would expect the relationship between reflex gain and specd of locomotion to be identical, regardless of the type of locomotion. The reflex gains during walking and running would fall along the same regression line, which was clearly not the case (Fig. 3). Substantial differences in the reflex gain were found between walking and running, for which we could not account by either the speed of locomotion or the motor output (i.e., EMG pattern).

Why the 2 forms of locomotion require different reflex gains is not certain, although some possibilities may be suggested. A primary difference between the 2 tasks is the proportion of time spent in the stance versus the swing phase. By definition, the stance phase must occupy less than $50 \%$ of the total step cycle time during running, and more than $50 \%$ during walking. This means that even when these 2 tasks are performed at the same speed of forward progression, the time spent in the stance phase is substantially shorter for running. Moreover, the requirement of a flight phase (i.e., when both feet are off the ground) in running necessitates a greater vertical excursion of the total body. These 2 factors (a shorter stance phase and a greater vertical displacement) together make for a more impulsive impact at foot contact and a more powerful push-off in late stance. The more rapid and forceful nature of both these events may lead to more synchronous firing of afferents. Muscle spindle sensory fibers are very sensitive to velocity, so the more rapid movements could lead to large reflex responses, which could disturb rather than aid the forward progression during running. A lower reflex gain may also be necessary to prevent saturation of the reflex pool.

Within a particular form of locomotion, the reflex gain is negatively correlated with both the background activation level of the homonymous muscle and the speed of locomotion (Fig. 3 ). This correlation is much higher for walking than for running, again highlighting differences between these 2 tasks. Because the speed of locomotion and the SOL EMG are highly correlated, these 2 factors could not be separated. In either case, the behavior of the reflex gain is consistent with the possibility that it is lowered in proportion to the background activation level or locomotion speed in order to prevent saturation of the motoneuron pool (Capaday and Stein, 1987a). If the afferent input from the muscle spindles contributes in an important way to locomotor activity of the SOL muscle, preventing saturation of this pathway would be essential.

\section{How are these task-dependent reflex gains activated?}

The H-reflex gain of the SOL muscle was shown to change rapidly from the high levels during standing to the low levels of walking. Moreover, the reflex gain of the first step was not different from that of the later steps, suggesting that conditions unique to the first step did not modify the reflex gain in a special way. The reflex depression seen in the first step was complete in that the reflexes were indistinguishable from those of steady 


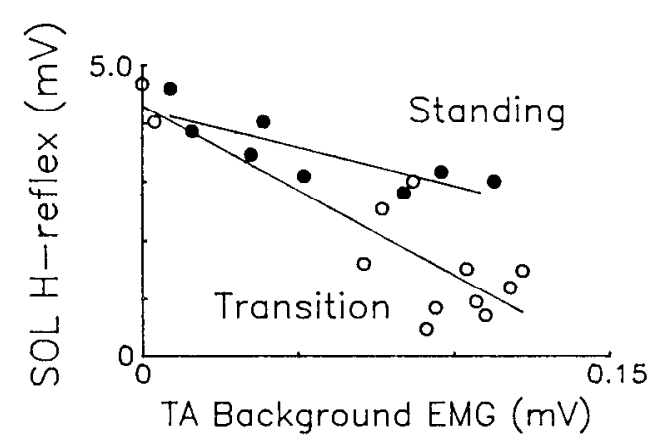

Figure 8. SOL H-reflex varied inversely $(r=-0.86$, slope $=-29.0)$ with activation level of TA in transition from standing to walking (open circles). These activation levels observed in the transition task were matched under static cocontraction (filled circles). An inverse relationship between the SOL H-reflex and the TA EMG was again observed, but to a lesser extent $(r=-0.82$, slope $=-13.2)$. The slopes of these 2 regression lines are significantly different at the $p<0.05$ level ( $t$ test).

state walking (Fig. 6). Apparently, the reflex gain of the SOL is switched immediately into the appropriate levels for walking at the time walking is initiated.

The gain change occurred within a reaction time and was coincident with the activation of the TA. This suggests that the central command to initiate walking affected the prime mover (TA) and the reflex gain of its antagonist (SOL) simultancously. A similar depression of the SOL H-reflex with activation of the TA has heen ohserved in single joint movements (Gottlieb et al., 1970) and more recently in a postural adjustment task (Yamashita and Moritani, 1989). Two possible mechanisms might be proposed to explain the decreased reflex: postsynaptic or presynaptic inhibition. For example, the central command to initiate walking may project to the TA motoneurons and their Ia inhibitory interneurons in parallel, as has been shown for several descending systems such as the corticospinal tract (Baldissera et al., 1981). The reflex depression would then reflect the postsynaptic effects of the Ia inhibitory interneurons on the SOL motoneuron pool. Alternatively, descending systems or spinal mechanisms could produce presynaptic effects on the Ia sensory terminals.

If the first possibility were true, the TA motoneurons would be activated in parallel with their Ia inhibitory interncurons, and one would expect the SOL EMG to vary in a reciprocal fashion to the TA. The H-reflex amplitude would then simply vary with the SOL activation pattern. This was clearly not the case. The SOL EMG pattern did not always vary reciprocally with the TA (e.g., Subject \#1 in Fig. 5) and the time course of the H-reflex modulation was independent of the SOL EMG in some subjects (e.g., Subject \#2 in Fig. 5).

Alternatively, the reciprocal effects from the TA may be acting presynaptically on the muscle spindle afferents from the SOL. This mechanism has been suggested previously (Tanaka et al., 1974), although evidence for its existence remains indirect. The pre- and postsynaptic effects of reciprocal inhibition were separated by static cocontraction experiments. Subjects maintained a constant SOL EMG level coupled with varying degrees of TA activation. The data of Figure 8 suggest that reciprocal inhibition associatcd with the activation of the antagonist also acts presynaptically on the agonist Ia afferents. The amplitude of the reciprocal effect was smaller under static conditions than during the dynamic conditions of walking, suggesting that the presynaptic effects may have phasic as well as tonic components.
Computer simulation (Capaday and Stein, 1987b) and tests in decerebrate cats (Capaday and Stein, 1989) have shown that various mixtures of postsynaptic excitation and inhibition leading to the same level of EMG activity have little effect on the level of the H-reflex. Thus, the task-dependent changes in H-reflex gain observed here suggest that presynaptic inhibition correlated to the activity in TA is involved (Fig. 7). Some controversy remains regarding the possibility of altering the gain of the motoneuron pool by postsynaptic factors (Kernell and Hultborn, 1990). The postsynaptic mechanisms that arc most cffective in altering the gain of the motoneuron pool are those that generate synaptic inputs that are systematically different from the normal order of small to large. Synaptic inputs that exert this type of differential effect on the motoneuron pool may also generate reversals in recruitment order. Reversals in motor unit recruitment order have not been shown in locomotion (Hoffer et al., 1987; Jakobssen et al., 1988), so we favor the explanation that presynaptic mechanisms alter the reflex gain in locomotion.

Future experiments will be needed to determine whether there are indeed separate mechanisms to control the time-dependent modulation of the reflex that is characteristic of all forms of rhythmic movement (Forssberg et al., 1977; Akazawa et al, 1982; Lund and Olsson, 1983; Mackay et al., 1983; Capaday and Stein, 1987a; Collins et al., 1989) and the different reflex gains that characterize different movement tasks (Capaday and Stein, 1986, 1987a).

\section{References}

Akazawa K, Aldridge JW, Steeves JD, Stein RB (1982) Modulation of stretch reflexes during locomotion in the mesencephalic cat. J Physiol 329:553-567.

Baldissera F, Hultborn H, Illert M (1981) Integration in spinal neuronal systems. In: Handbook of physiology, Section I, Vol II, Motor Control, Part I (Brooks VB, ed), pp 509-595. Bethesda: American Physiological Society.

Boorman GI, Becker WJ, Lee RG (1989) Modulation of the H-reflex during stationary cycling in normal humans and patients with spinal spasticity. Soc Neurosci Abstr, Vol 15, Part I, p 691.

Capaday C, Stein RB (1986) Amplitude modulation of the soleus $\mathrm{H}$-reflex in the human during walking and standing. J Neurosci 6 : 1308-1313.

Capaday C, Stein RB (1987a) Difference in the amplitude of the human soleus H reflex during walking and running. J Physiol (Lond) 392: 513-522.

Capaday C, Stein RB (1987b) A method for simulating the reflex output of a motoneuron pool. J Neurosci Meth 21:91-104.

Capaday C, Stein RB (1989) The effects of postsynaptic inhibition on the monosynaptic reflex of the cat at different levels of motoneuron pool activity. Exp Brain Res 77:577-584.

Collins DF, Brooke JD, Mcllroy WE (1989) Inhibition of an homonymous monosynaptic, but not an heteronymous oligosynaptic, short latency reflex in the human leg during walking. Soc Ncurosci Abstr, Vol 15, Part II, p 1200.

Crenna P, Frigo C (1987) Excitability of the soleus H-reflex arc during walking and stepping in man. Exp Brain Res 66:49-60.

Dietz V, Faist M, Pierrot-Deseilligny E (1990) Amplitude modulation of the quadriceps H-reflex in the human during the early stance phase of gait. Exp Brain Res 79:221-224.

Feller W (1957) An introduction to probability theory and its applications, 2nd ed. New York: Wiley.

Forssberg H, Grillner S, Rossignol S (1977) Phasic gain control of reflexes from the dorsum of the paw during spinal locomotion. Brain Res 132:121-139.

Gottlieb GL, Agarwal GC, Stark L (1970) Interactions between voluntary and postural mechanisms of the human motor system. J Neurophysiol 33:365-381.

Hoffer JA, Loeb GE, Marks WB, O’Donovan MJ, Pratt CA, Sugano N (1987) Cat hindlimb motoneurons during locomotion. I. Destina- 
tion, axonal conduction velocity and recruitment threshold. J Neurophysiol 57:510-529.

Hoffmann P (1918) Uber die Beziehungen der Sehnenreflexe zur willkurlichen Bewegung und zum Tonus. Z Biol 68:351-370.

Jakobssen F, Borg K, Edstrom L, Grimby L (1988) Use of motor units in relation to muscle fiber type and size in man. Muscle Nerve 11: 1211-1218.

Kernell D, Hultborn H (1990) Synaptic effects on recruitment gain: a mechanism of importance for the input-output relations of motoneurone pools? Brain Res 507:176-179.

Llewellyn MGA (1989) Refiex and artificial control of muscle during locomotion. PhD thesis, St. Thomas' Hospital Medical School, University of London.

Lund JP, Olsson KA (1983) The importance of reflexes and their control during jaw movement. Trends Neurosci 6:458-463.
Mackay WA, Kwan HC, Murphy JT, Wong YC (1983) Stretch reflex modulation during a cyclic elbow movement. Electroenceph Clin Neurophysiol 55:687-698.

Myklebust BM, Gottlieb GL, Agarwal GC (1984) Orientation-induced artifacts in the measurement of monosynaptic reflexes. Neurosci Let 48:223-230.

Pedhazur EJ (1982) Multiple regression in behavioral research. New York: CBS College.

Tanaka R (1974) Reciprocal Ia inhibition during voluntary movements in man. Exp Brain Res 21:529-540.

Yamashita N, Moritani T (1989) Anticipatory changes of soleus H-reflex amplitude during execution process for heel raise from standing position. Brain Res 490:148-151. 